\title{
Computational Studies of Furanone and its 5Methyl/5Phenyl Derivatives
}

\author{
Nikoo Ghanbari ${ }^{1}$, Homa Azizian ${ }^{2}$, Mahmoud Mirzaei $^{3, 凶}$
}

Received: 08 March 2020 / Accepted: 09 March 2020 / Published Online: 16 March 2020

(C) SAMI Publishing Company (SPC) 2020

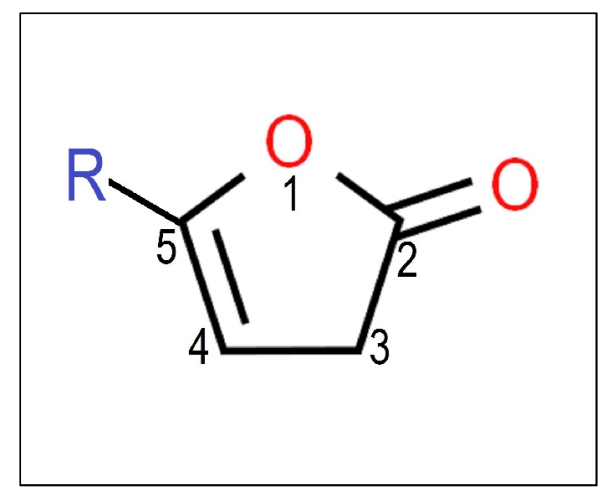

\begin{abstract}
The properties for 2(5H)-furanone and 2(5Methyl)- and 2(5Phenyl)furanone derivatives have been explored by computational chemistry approach. The subatomic unit calculations have been done to optimize the models and to evaluate their corresponding properties, in which several achievements have been seen for the investigated models. The energy levels of molecular orbitals indicated the importance of structural modifications for obtaining better electronic properties. To this aim, total energy, energy levels of the highest occupied and the lowest unoccupied molecular orbitals, energy gap, ionization positional, electron affinity, hardness, softness and dipole moment have been evaluated in addition to the original molecular weight and LogP parameters. The results revealed better reactivity and antioxidativity for 2(5Phenyl)furanone in comparison with two other models proposing it for various possible applications in biological systems. Moreover, hardness and softness properties were also seen more favorable for this model. As a conclusion, the importance of furanone could be very much increased regarding structural modification, which could be very well investigated by the computational chemistry approach.
\end{abstract}

Keywords: Furanone; Structural modification; Antioxidativity; Computational chemistry.

\section{Introduction}

Starting from small organic structures is an important way to synthesize complex organic or medicinal related compounds. Furanone (Fig. 1) is

\footnotetext{
$\triangle$ Corresponding author.

E-mail address: mdmirzaei@pharm.mui.ac.ir (M. Mirzaei)

${ }^{1}$ Faculty of Pharmaceutical Chemistry, Tehran Medical Sciences, Islamic Azad University, Tehran, Iran
}

among the most important mentioned small organic structures for various types of organic and bioorganic synthesis [1]. Furanone could be extracted from natural resources; however, its

\footnotetext{
2 Department of Medicinal Chemistry, School of PharmacyInternational Campus, Iran University of Medical Sciences, Tehran, Iran

${ }^{3}$ Biosensor Research Center, School of Advanced Technologies in Medicine, Isfahan University of Medical Sciences, Isfahan, Iran
} 
synthetic production has been also developed due to its large consumption in different chemical structure generations [2]. Based on the bioorganic purposes, several therapeutical activities have been seen for those generated structures from starting furanone including anticancer, analgesic, anticonvulsant, anti-inflammatory, antifungal, antibacterial, antimalarial, antioxidant, antimicrobial, antipsychotic, antiulcerative, antituberculosis, antiviral, vasorelaxant action and so many other applications [3-10]. The important role of furanone in therapeutics designated it as a famous pharmacophore for treatments of living systems [11]. Therefore, investigating its characteristic properties is still a formidable task to develop novel medicinal related compounds. Within this work, properties for $2(5 \mathrm{H})$-furanone heterocyclic structure (Fig. 1) has been investigated based computational chemistry approach. Furthermore, methyl and phenyl functionalization of $5 \mathrm{H}$ position have been considered for investigating the effects of structural modification on the properties of starting and resulted structures (Table 1). The molecular orbital properties could designate the properties such as reactivity and antioxidativity very well, in which computational chemistry approach are almost the best technique to evaluate them as employed by earlier works for different theoretical and experimental purposes [12]. Structural modifications are important to generate new structures from the starting one; however, the physicochemical properties should be tracked to see what will happen after modification. To this aim, molecular properties have been evaluated for the original furanone and two of $5 \mathrm{H}$ derivatives to explore the effects of structural modifications in addition to molecular orbital properties of new structures (Table 1). Knowledge about subatomic unites properties for chemical structures could help to provide new structures based on desired proposes, which are very much important to introduce new compounds especially for treatments of living systems [13-25].

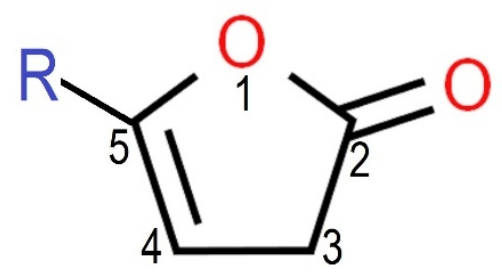

Fig. 1. 2(5R)-Furanone; $\mathrm{R}=H$, Methyl or Phenyl.

\section{Materials and Methods}

Four steps have been designed to perform the computational processes to obtain the required properties for achieving the goals of this work. First, the 3D molecular models of $2(5 \mathrm{H})$-furanone (F), 2(5Methyl)-furanone (5MF) and 2(5Phenyl)furanone (5PF) and the values of molecular weight $(M W)$ and $\log \mathrm{P}$ have been obtained from the ChemSpider structural bank [26]. Second, all obtained 3D molecules have been optimized to reach their minimum energy structures based on density functional theory (DFT). To this aim, the B3LYP exchange-correlation functional and the 631G* standard basis set have been employed to perform DFT calculations by the Gaussian package of program [27]. Third, the molecular descriptors including total energy $\left(E_{\mathrm{T}}\right)$, energies of the highest occupied and the lowest unoccupied molecular orbitals $\left(E_{\mathrm{H}}\right.$ and $\left.E_{\mathrm{L}}\right)$ and dipole moment $(D M)$ have been directly extracted from the calculation results in addition to the stabilized structures. Fourth, further molecular descriptors including energy gap $\left(E_{\mathrm{G}}\right)$, ionization potential $(I)$, electron affinity $(A)$, hardness $(H)$ and softness $(S)$ have been evaluated using the eqs. (1) - (5) [28].

$$
\begin{aligned}
& E_{G}=E_{\mathrm{L}}-E_{\mathrm{H}} \\
& I=-E_{\mathrm{H}} \\
& A=-E_{\mathrm{L}} \\
& H=E_{\mathrm{G}} / 2 \\
& S=1 / H
\end{aligned}
$$

All the investigated models description including the models representation and molecular descriptors are exhibited in Table 1. It is important 
to note that the computational chemistry could provide insightful information about the characteristic properties of chemical structures in both of singular and complex systems [29]. Quantum mechanical (QM) and molecular mechanical (MM) methods have been developed very well to investigate almost all chemical structures and processes prior to or parallel with experiments. Molecular graphics and computational softwares could provide the in silico environment for high-performance research activities [30-40]. By these benefits, this work has been done to carefully investigate the characteristic models description of $F, 5 \mathrm{MF}$ and 5PF structures based computational chemistry approach.

Table 1: Models description*

\begin{tabular}{llll}
\hline Name & $2(5 H)$-Furanone & $2(5$ Methyl)-Furanone \\
Symbol & $\mathrm{F}$ & $5 \mathrm{MF}$ & 2 (5Phenyl)-Furanone \\
CSID & 124157 & 11071 & \\
Figures & & \\
& &
\end{tabular}

"Please see Fig. 1 for starting model.

\section{Results and discussion}

The models of this work include F, 5MF and 5PF, in which the starting structure is shown in Fig. 1 and details are listed in Table 1. As mentioned earlier, the structures have been optimized to reach the minimum energy structures to obtain their properties in addition the stabilized structures. Comparing the molecular weights $(\mathrm{MW})$ of three compounds shows than we have three levels of structures, in which $\mathrm{F}$ is the lightest and $5 \mathrm{PF}$ is the heaviest one, with 5MF in the middle. The obtained values of $\log \mathrm{P}$, the solvation proportion of substance in oil/water media, show that the solubility of functionalized structures is leading to oil media for heavier structures. It is noted that the (C) SAMI Publishing Company (SPC)
$5 R$ position of furanone (Fig .1) is an important atomic site for structural modification, in which the structures could be stabilized and their properties could be evaluated. Looking at the results of Table 1 indicates that the values of total energies $\left(E_{T}\right)$ are changed for the investigated models, which is reasonable because of different numbers of atoms and their corresponding subatomic unites. Moreover, the molecular orbital properties $\left(E_{\mathrm{H}}\right.$ and $\left.E_{\mathrm{L}}\right)$ also indicate the effects of structural modifications on the electronic environments of corresponding structures. In addition to the exact values of $E_{\mathrm{H}}$ and $E_{\mathrm{L}}$, their energy gaps $\left(E_{\mathrm{G}}\right)$ show the level of reactivity, in which the most reactive one could be designated 
for $5 \mathrm{PF}$ with lower value of $E_{\mathrm{G}}$. It is important to note here that the energy levels of the highest occupied and the lowest unoccupied molecular orbitals (HOMO and LUMO) are crucial for electronics of chemical structures, in which the electron $s$ should be transferred into these levels to contribute to chemical reactions. Therefore, ionization potential and electron affinity ( $I$ and $A$ ) could be defined based on the values of HOMO and LUMO. Regarding the obtained results, better $I$ and $A$ properties have been obtained for 5PF in comparison with both of $\mathrm{F}$ and $5 \mathrm{MF}$ in agreement with the achievement on chemical reactivity. For antioxidant activity, the values of $I$ and $A$ are dominant to propose a chemical structure for further use in the aspects of antioxidativity. These antioxidant agents could play important roles for inhibiting the growth of cancer cells. Within current results, these properties could be seen by the structural modifications to achieve better activities. Considering no target, structural properties of chemical substances could lead them to biological activities for drug design purposes, which is called ligand-based drug design (LBDD) [41]. To this aim, computational chemistry could provide in silico environment for drug design processes [42-49]. In agreement with the results up to now, hardness and softness ( $H$ and $S$ ) also indicate better properties for 5PF in comparison with $\mathrm{F}$ and $5 \mathrm{MF}$, which reveals that $5 \mathrm{PF}$ could be employed for the reactivity purposes better than other two models. Lower value of $H$ and higher value of $S$ for $5 P F$ indicate better situation for contributing to chemical reactivity, which is an important property for various applications,

\section{References}

1. Luo SH, Yang K, Lin JY, Gao JJ, Wu XY, Wang ZY. Synthesis of amino acid derivatives of 5-alkoxy-3, 4dihalo-2 (5H)-furanones and their preliminary bioactivity investigation as linkers. Org. Biomol. Chem. 2019;17:5138-5147.

2. Schwab W. Natural 4-hydroxy-2, 5-dimethyl-3(2H)furanone (Furaneol ${ }^{\circledR}$ ). Molecules 2013;18:6936-6951. especially in antioxidativity. The obtained values of dipole moments $(D M)$ show different electronic balance in the investigated structures.

\section{Conclusion}

Computational chemistry approach has been employed within this work to investigate properties for furanone and its methyl and phenyl derivatives. The structural modification could significantly change the properties of structures regarding their subatomic unit calculations. The results indicated that the chemical reactivity of structures are changed based on the employed modifications, in which phenyl group plays a dominant role to evaluate characteristic properties for functionalized furanone. Moreover, the energy levels of molecular orbitals indicated that electron transfer properties could be better achieved for phenyl functionalized furanone in comparison with the original furanone and methyl functionalized one. Antioxidativity activity of furanone could be increased by phenyl functionalization, in addition to better activity. The oil/water solubility is also increased for the phenyl functionalized structure, in which this property is crucial for drug design processes. Finally, the properties of investigated structures have been very well evaluated based on the employed computational chemistry approach.

\section{Acknowledgements}

The authors acknowledge the supports of this work by the research council of Islamic Azad University under the grant number 70ShD, which has been done as the master thesis of N. Ghanbari.

3. Kozminykh VO, Igidov NM, Kozminykh EN, Konshina LO, Semenova ZN, Lyadova NV, Plaksina AN, Andreichikov YS. Synthesis and antimicrobial activity of 2substituted-5-aryl-2,3-dihydro-3-furanones. Pharm. Chem. J. 1991;25:891-897.

4. Cannon GW, Breedveld FC. Efficacy of cyclooxygenase2 specific inhibitors. Am. J. Med. 2001;3: 6-12. 
5. Ogara JP, Humphreys $H$, Staphylococcus epidermidis biofilms importance and implications, J. Med. Microbiol. 2001;50:582-587.

6. Futaki N, Yoshikawa K, Hamasaka Y, Arai I, Higuchi S, lizuka $\mathrm{H}$, Otomo S. A novel non-steroidal antiinflammatory drug with potent analgesic and antipyretic effects, which causes minimal stomach lesions. Gen. Pharmacol. 1997;24:105-110.

7. Razet $R$, Thomet $U$, Furtmuller $R$, Chiaroni $A$, Sigel $E$, Sieghart W, Dodd RH. 5-[1-(2-N-arylsulfonyl-1,2,3,4tetrahydroisoquinolyl)]-4,5dihydro-2(3H)-furanones: positive allosteric modulators of the GABA receptor with a new mode of action. J. Med. Chem. 2000;43:4363-4366.

8. Abou-Elmagd WSI, Hashem Al. Synthesis and antitumor activity evaluation of some novel fused and spiro heterocycles derived from a $2(3 \mathrm{H})$-furanone derivative, J. Heterocycl. Chem. 2016;53: 202-208.

9. Akhter M, Saha R, Tanwar O, Alam MM, Zaman MS. Synthesis and antimalarial activity of quinolinesubstituted furanone derivatives and their identification as selective falcipain-2 inhibitors. Med. Chem. Res. 2015;24:879-890.

10. Wang Y, Gloer JB, Scott JA, Malloch D, Appenolides AC. Three new antifungal furanones from the coprophilous fungus Podospora appendiculata. J. Nat. Prod. 1993;56:341-344.

11. Husain A, Khan SA, Iram F, Iqbal MA, Asif M. Insights into the chemistry and therapeutic potential of furanones: A versatile pharmacophore. Eur. J. Med. Chem. 2019;171:66-92.

12. Mardirossian N, Head-Gordon M. Thirty years of density functional theory in computational chemistry. Mol. Phy. 2017;115:2315-2372.

13. Mirzaei M, Hadipour NL. Study of hydrogen bonds in crystalline 5-nitrouracil. Density functional theory calculations of the 0-17, N-14, and $\mathrm{H}-2$ nuclear quadrupole resonance parameters. J. Iran. Chem. Soc. 2009;6:195-199.

14. Mirzaei M, Mirzaei M. The C-doped AIP nanotubes: A computational study. Solid State Sci. 2011;13:244-250.

15. Behzadi H, Hadipour NL, Mirzaei M. A density functional study of $170,14 \mathrm{~N}$ and $2 \mathrm{H}$ electric field gradient tensors in the real crystalline structure of $\alpha$ glycine. Biophys. Chem. 2007;125:179-183.

16. Mirzaei M, Yousefi M. Computational studies of the purine-functionalized graphene sheets. Superlat. Microstruct. 2012;52:612-617.

17. Samadi Z, Mirzaei M, Hadipour NL, Khorami SA. Density functional calculations of oxygen, nitrogen and hydrogen electric field gradient and chemical shielding tensors to study hydrogen bonding properties of peptide group (OC-NH) in crystalline acetamide. J. Mol. Graph. Model. 2008;26:977-981.

18. Mirzaei M. Effects of carbon nanotubes on properties of the fluorouracil anticancer drug: DFT studies of a CNT-fluorouracil compound. Int. J. Nano Dimens. 2013;3:175-179.

19. Partovi T, Mirzaei M, Hadipour NL. The $\mathrm{C}-\mathrm{H} \cdots \mathrm{O}$ hydrogen bonding effects on the 170 electric field gradient and chemical shielding tensors in crystalline 1methyluracil: A DFT study. Z. Naturforsch. A. 2006;61:383-388.

20. Harismah K, Sadeghi M, Baniasadi R, Mirzaei M. Adsorption of vitamin $C$ on a fullerene surface: DFT studies. J. Nanoanal. 2017;4:1-7.

21. Mokhtari A, Harismah K, Mirzaei M. Covalent addition of chitosan to graphene sheets: Density functional theory explorations of quadrupole coupling constants. Superlat. Microstruct. 2015;88:56-61.

22. Harismah K, Ozkendir OM, Mirzaei M. Explorations of crystalline effects on 4-(benzyloxy) benzaldehyde properties. Z. Naturforsch. A. 2015;70:1013-1018.

23. Mirzaei $M$, Harismah $K$, Jafari $E$, Gülseren $O$, Rad AS. Functionalization of $(n, 0)$ CNTs $(n=3-16)$ by uracil: DFT studies. Eur. Phys. J. B. 2018;91:14.

24. Harismah K, Mirzaei M, Sahebi H, Gülseren O, Rad AS. Chemically uracil-functionalized carbon and silicon carbide nanotubes: Computational studies. Mater. Chem. Phys. 2018;205:164-170.

25. Harismah K, Mirzaei M, Ghasemi N, Nejati M. Noncovalent functionalisation of $\mathrm{C} 30$ fullerene by pyrrole$n$-carboxylic acid $(n=2,3)$ : Density functional theory studies. Z. Naturforsch. A. 2017;73:51-56.

26. Pence HE, Williams A. ChemSpider: An online chemical resource. J. Chem. Edu. 2010;87:1123-1124.

27. Frisch MJ, Trucks GW, Schlegel HB, Scuseria GE, Robb MA, Cheeseman JR, Zakrzewski VG, Montgomery Jr JA, Stratmann RE, Burant JC, Dapprich S, et al. Gaussian 98, Revision A. 7. Pittsburgh, PA: Gaussian. Inc. Computer Program. 1998.

28. Aramideh M, Mirzaei M, Khodarahmi G, Gülseren O. DFT Studies of Graphene-Functionalised Derivatives of Capecitabine. Z. Naturforsch. A. 2017;72:1131-1138.

29. Aghazadeh $M$, Mirzaei $M$. Hydrogen bond interactions in sulfamerazine: DFT study of the $0-17, \mathrm{~N}-14$, and $\mathrm{H}-2$ electric field gradient tensors. Chem. Phys. 2008;351:159-162.

30. Soleimani M, Mirzaei M, Mofid MR, Khodarahmi G, Rahimpour SF. Lactoperoxidase inhibition by tautomeric propylthiouracils. Asian J. Green Chem. 2020;4:1-0. 
31. Alidoosti ZS, Mirzaei M. Comparative examination of moclobemide, tranylcypromine, phenelzine and isocarboxazid for monoamine oxidase-A inhibition. Adv. J. Chem. B. 2019;1:23-28.

32. Esfahani AN, Mirzaei M. Flavonoid derivatives for monoamine oxidase-A inhibition. Adv. J. Chem. B. 2019;1:17-22.

33. Ozkendir OM, Mirzaei M. Alkali metal chelation by 3hydroxyl-4-pyridinone. Adv. J. Chem. B. 2019;1:10-6.

34. Nazemi H, Mirzaei M, Jafari E. Antidepressant activity of curcumin by monoamine oxidase-A inhibition. J. Adv. Chem. B. 2019;1:3-9.

35. Mirzaei M, Meskinfam M. Computational studies of effects of tubular lengths on the NMR properties of pristine and carbon decorated boron phosphide nanotubes. Solid State Sci. 2011;13:1926-1930.

36. Mirzaei M. Calculation of chemical shielding in C-doped zigzag BN nanotubes. Monatsh. Chem. 2009;140:12751278.

37. Bagheri Z, Mirzaei M, Hadipour NL, Abolhassani MR. Density functional theory study of boron nitride nanotubes: calculations of the N-14 and B-11 nuclear quadrupole resonance parameters. J. Comput. Theor. Nanosci. 2008;5:614-618.

38. Mirzaei M, Hadipour NL, Abolhassani MR. Influence of C-doping on the B-11 and N-14 quadrupole coupling constants in boron-nitride nanotubes: A DFT study. $Z$. Naturforsch. A 2007;62:56-60.

39. Mirzaei M, Mirzaei M. The B-doped SiC nanotubes: A computational study. J. Mol. Struct. THEOCHEM 2010;953:134-138.

40. Mirzaei M, Hadipour NL, Ahmadi K. Investigation of C$\mathrm{H} . . . \mathrm{O}=\mathrm{C}$ and $\mathrm{N}-\mathrm{H} . . . \mathrm{O}=\mathrm{C}$ hydrogen-bonding interactions in crystalline thymine by DFT calculations of 0-17, N-14 and H-2 NQR parameters. Biophys. Chem. 2007;125:411-415.

41. Acharya C, Coop A, E Polli J, D MacKerell A. Recent advances in ligand-based drug design: relevance and utility of the conformationally sampled pharmacophore approach. Cur. Comput. Aided Drug Design. 2011;7:10-22.

42. Mirzaei M, Hadipour NL. A computational NQR study on the hydrogen-bonded lattice of cytosine-5-acetic acid. J. Comput. Chem. 2008;29:832-838.

43. Mirzaei M, Hadipour NL, Seif A, Giahi M. Density functional study of zigzag $\mathrm{BN}$ nanotubes with equivalent ends. Physica E 2008;40:3060-3063.

44. Mirzaei M. Density functional study of defects in boron nitride nanotubes. Z. Phys. Chem. 2009;223:815-823.

45. Mirzaei M. A computational NMR study of boron phosphide nanotubes. Z. Naturforsch. A 2010;65:844848.

46. Harismah K, Mirzaei M, Moradi R. DFT studies of single lithium adsorption on coronene. Z. Naturforsch. A 2018;73:685-691.

47. Mirzaei M, Hadipour NL. Study of hydrogen bonds in 1methyluracil by DFT calculations of oxygen, nitrogen, and hydrogen quadrupole coupling constants and isotropic chemical shifts. Chem. Phys. Lett. 2007;438:304-307.

48. Mirzaei M. Carbon doped boron phosphide nanotubes: a computational study. J. Mol. Model. 2011;17:89-96.

49. Harismah K, Mirzaei M, Samadizadeh M, Rad AS. DFT studies of stabilities and properties for X3Y6Z9 borazine-like structures $(X=B / A l, Y=N / P, Z=H / M e)$. Superlat. Microstruct. 2017;109:360-365. 5Methyl/5Phenyl Derivatives. Adv. J. Chem. B. 2020;2(1):33-38. doi: 10.33945/SAMI/AJCB.2020.1.6 